\title{
Elaboração e validação de cartilha sobre cuidados com o prematuro no processo de
}

\section{alta hospitalar}

\author{
Elaboration and validation of a primer about care for premature babies in the hospital discharge
}

\section{process}

Elaboración y validación de una cartilla sobre el cuidado de bebés prematuros en el proceso de alta hospitalaria

\section{Resumo}

Objetivo: construir e validar uma cartilha educativa ilustrada para familiares sobre cuidados com o prematuro no processo de alta da Unidade de Terapia Intensiva Neonatal. Metodologia: estudo metodológico, com abordagem quantitativa. Para o ajuizamento participaram 13 especialistas e 20 responsáveis de bebês ou crianças de até um ano nascidas prematuras, por meio de formulários. Foi utilizada a escala tipo Likert e o Índice de Validade de Conteúdo para avaliar e validar, respectivamente, conforme modelo de Pasquali. Resultados: organizados em: elaboração de cartilha, validação e adequação do manual educativo com juízes especialistas e público-alvo. O conhecimento e sugestões de cada público foram essenciais para tornar legítima e eficaz os temas propostos na cartilha. Conclusão: a cartilha educativa foi construída e validada alcançando índices satisfatórios. O material contém orientações que servirão de suporte para a educação em saúde.

Palavras-chave: Recém-nascido prematuro; Alta do paciente; Unidade de Terapia Intensiva Neonatal; Educação em saúde.

\begin{abstract}
Objective: to build and validate an illustrated educational booklet for family members about care for the premature baby in the discharge process from the Neonatal Intensive Care Unit. Methodology: the participants in this qualitative, methodological study were thirteen experts and twenty responsible of babies or children up to one year of age, using online forms. A Likert scale and the Content Validity Index were used to evaluate and validate, respectively. Approved by the Research Ethics Committee. Results: the data were organized in: preparation of booklet; and, validation and adequacy of the educational manual with expert judges and target audience. The knowledge and suggestions of each audience were essential to make the themes proposed in the booklet legitimate and effective. Conclusion: an educational booklet was built and validated, achieving satisfactory rates. The material contains orientations that will help health education.
\end{abstract}

Keywords: Premature; Patient discharge; Intensive Care Units; Health education. 


\section{Resumen}

Objective: construir y validar un folleto educativo ilustrado para miembros de la familia sobre el cuidado del bebé prematuro en el proceso de alta de la Unidad de Cuidados Intensivos Neonatales. Metodología: el estudio metodológico, con un enfoque cualitativo, involucró a 13 especialistas y 20 gerentes de bebés o niños de hasta un año de edad, nacidos prematuramente, a través de formularios en línea. La escala Likert y el índice de validez de contenido se utilizarán para evaluar y validar, respectivamente. Aprobado por el Comité de Ética de Investigación. Resultados: te organizas en: preparar el folleto; y validación y adaptación del manual educativo con especialistas y audiencias públicas. El conocimiento y las sugerencias de cada audiencia son esenciales para que los temas propuestos en el folleto sean legítimos y efectivos. Conclusión: la tarjeta educativa fue construida y validada, alcanzando tasas satisfactorias.

Palabras clave: Recien nacido prematuro; Alta del paciente; Unidades de Cuidado Intensivo Neonatal; Educácion en salud.

\section{Introdução}

O nascimento prematuro, aquele com idade gestacional menor que 37 semanas, está entre as principais causas de internação em Unidades de Terapia Intensiva Neonatal (UTIN) e responde por aproximadamente um terço dos casos de óbitos neonatais (Teixeira, et al., 2019).

O cuidado adequado ao recém-nascido pré-termo tem sido um dos desafios para reduzir os índices de morbimortalidade infantil no país, visto que as comorbidades respiratórias, cardíacas, neurológicas e infecciosas, mesmo sendo as mais prevalentes na prematuridade e principais causadores de morte em menores de cinco anos, podem ser preveníveis com cuidados de alta qualidade no período do pré-natal, durante o parto e imediatamente após o nascimento (Teixeira, et al., 2019; World Health Organization, 2018).

As UTIN ao longo dos anos vêm se modernizando, sendo equipadas com tecnologia de ponta, contribuindo para uma assistência mais efetiva ao recém-nascido de risco e melhoria da sua sobrevida, tendo principalmente como foco aspectos biológicos da assistência. Contudo, levando em consideração esse ambiente cercado de profissionais, luzes, ruídos, tecnologias e rotinas duras, a hospitalização aliada ao nascimento prematuro da criança se torna um processo angustiante e doloroso aos pais, que pode ocasionar danos físicos e cognitivos, além de sentimentos como medo, perda e luto (Estevam \& Silva, 2016; Fialho, Dias, Silva, Santos \& Salvador, 2015; Lelis, et al., 2018).

Diante desse cenário, já vem sendo debatido frequentemente entre as instituições de saúde e os profissionais de saúde o fortalecimento de ações que envolvem a humanização e o uso de tecnologia relacional que priorize a assistência efetiva à tríade pais-filho-família. Dentre tais ações estão a inserção e participação dos pais no cuidado ao bebê, bem como o planejamento da alta hospitalar durante toda a internação, a fim de estimular e fortalecer o vínculo entre pais-bebê e conferir segurança e autonomia para garantir continuidade do cuidado no domicílio, de modo a contribuir para a redução da morbimortalidade neonatal (Alcântara, et al., 2017; Brasil, 2014; Klock, Buscher, Erdmann, Costa \& Santos, 2019; Lelis, et al., 2018; Rodrigues, Uema, Rissi, Felipin \& Higarashi, 2019; Sousa, Medino, Benevides, Ibiapina \& Ataíde, 2019).

Entretanto, além da inclusão da família nos cuidados, o diálogo durante todo o período da internação e aumento do vínculo família-profissional também são ferramentas essenciais no preparo de alta de familiares de recém-nascidos hospitalizados na UTIN. O processo de alta hospitalar do prematuro pode gerar grande preocupação aos familiares, levando em consideração a complexidade do cuidado que muitas dessas crianças demandam e também a falta de saberes e habilidades específicas de muitos responsáveis diante dessa nova situação (Marski, Custodio, Abreu, Melo \& Wernet, 2016).

A participação dos pais dentro da UTIN se torna limitada tanto pela fragilidade clínica do bebê, quanto pela dificuldade de comunicação dos profissionais de saúde, devido principalmente à escassez de enfermeiros e sua sobrecarga. Além disso, o desencontro de informações instruídas aos familiares por diferentes profissionais é um fator que gera sentimento de insegurança (Alcântara, et al., 2017; Silva, Gomes, Marta, Araujo \& Braga, 2020). 
Sendo assim, uma boa alternativa como alternativa para minimizar esta lacuna é o desenvolvimento e implementação de recursos de apoio à educação em saúde, tais como cartilhas e manuais educativos, a fim de melhorar o entendimento, a adesão às orientações e auxiliar no autocuidado de pacientes, sendo úteis no processo de alta hospitalar do bebê prematuro (Silva, et al., 2018).

Diante dessa perspectiva, o objetivo do estudo foi construir e validar uma cartilha educativa ilustrada para familiares sobre cuidados com o prematuro no processo de alta da UTIN.

\section{Metodologia}

Trata-se de estudo metodológico, realizado de janeiro de 2019 a maio de 2020, no qual foram desenvolvidos três conjuntos de procedimentos para validação de conteúdo, conforme modelo de Pasquali, a saber, teóricos, empíricos e analíticos (Medeiros, et al., 2015). As etapas seguidas foram: 1) Elaboração da cartilha; 2) Validação do material educativo com juízes especialistas e público-alvo; 3) Adequação da cartilha educativa.

As etapas do âmbito teórico foram constituídas da busca na literatura dos principais temas para composição da cartilha, do estudo desses temas de modo a constituir um referencial conceitual e da elaboração da cartilha educativa com conteúdo textual e ilustrativo.

Quanto às etapas do âmbito empírico prosseguiu-se à coleta de dados para avaliação, aprimoramento e validação da cartilha. Utilizou-se como estratégia a geração de um link pela ferramenta Google Docs ${ }^{\circledR}$ de modo a direcionar o respondente a uma carta convite, seguida do Termo de Consentimento Livre e Esclarecido (TCLE), no qual mediante a concordância ocorria uma direcionamento para uma página com a cartilha construída em formato PDF e em seguida, a um questionário de avaliação composto de uma caracterização do respondente e uma escala tipo Likert para os seguintes aspectos: clareza da linguagem, coerência, pertinência e estrutura, com um item ao final livre para sugestões. A valoração da escala para cada item foi: (1) Discordo totalmente, (2) Discordo, (3) Indeciso, (4) Concordo, (5) Concordo totalmente.

Vale destacar que houve adaptação da linguagem para atender aos dois grupos de participantes pretendidos no estudo: enfermeiros juízes; e pais ou responsáveis de bebês prematuros. Foi especificado ainda o prazo de até sete dias a partir da data de envio para a devolução da análise do material educativo.

A captação dos participantes do estudo se deu mediante a técnica bola de neve, tendo como cenário de partida, para a captação de enfermeiros juízes, um grupo de pesquisa na área de enfermagem materno-infantil de uma Universidade Pública, na cidade do Rio de Janeiro. A escolha deste cenário se deu por ser este um espaço que congrega docentes e enfermeiros especialistas na área pediátrica e neonatal.

O contato de apresentação da pesquisa foi feito através de mensagem por WhatsApp® ao referido grupo, mediante autorização da coordenação. Os primeiros enfermeiros juízes captados, considerados 'sementes' indicaram novos participantes, seguindo-se uma cadeia de referência (Vinuto, 2014). Foi realizado o envio, individual, do link para avaliação da cartilha para os enfermeiros que preenchiam os critérios de inclusão: ser enfermeiro atuante na área da assistência ou docência, por no mínimo dois anos, no ensino em saúde do recém-nascido (RN) ou em assistência em UTIN. Nesta etapa de captação dos participantes, não houve recusas nem desistências quanto à participação.

Uma segunda etapa de validação foi realizada com o público-alvo da cartilha educativa, envolvendo pais ou responsáveis, maiores de 18 anos, cujos filhos tivessem nascidos prematuros, necessitaram de hospitalização em UTIN e estivessem com até um ano de idade no período da coleta de dados. A captação desses responsáveis também seguiu uma cadeia de referência a partir da indicação de enfermeiros do referido grupo de pesquisa. Assim, participaram 13 especialistas e 20 responsáveis de bebês ou crianças de até um ano nascidas prematuras. 
Visando ao anonimato dos integrantes, o nome dos enfermeiros juízes foi substituído pela letra $\mathbf{J}$ e para os pais ou responsáveis participantes utilizando a letra P. Em seguida, colocou-se o número correspondente a ordem de execução das entrevistas.

Por fim, para as etapas do âmbito analítico utilizou-se o Índice de Validade de Conteúdo (IVC) que é calculado utilizando-se o somatório das respostas concordo (4) e concordo totalmente (5), dividido pelo número total de respostas para o item. Foram considerados válidos os itens que alcançaram porcentagem de concordância entre os participantes, com um nível pré-estabelecido de no mínimo 80\%, conforme a literatura recomenda (Sousa, Turrini \& Poveda, 2015).

Após cada etapa de coleta foram realizados ajustes na cartilha de modo a atender sugestões julgadas pertinentes para a melhoria do material educativo no que tange ao conteúdo e ilustrações.

O projeto do estudo foi aprovado pelo Comitê de Ética e Pesquisa da Universidade Federal do Estado do Rio de Janeiro, de acordo com a Resolução nº 466/2012, sob o parecer 3.564.672.

\section{Resultados}

Participaram deste estudo 13 (100\%) enfermeiros juízes do sexo feminino, com dois anos ou mais de atuação no ensino em saúde do recém-nascido ou em assistência em UTIN. Sendo que destes, 9 (69,3\%) eram especialistas em Enfermagem Neonatal, 3 (23\%) em Enfermagem Neonatal e Pediátrica e 1 (7,7\%) em Enfermagem Pediátrica. No que se refere ao local de atuação, 6 (46\%) eram da assistência em UTIN, 3 (23\%) da docência (ensino em saúde do recém-nascido), 1 $(7,7 \%)$ atua em ambos os locais, 2 (15,6\%) em Banco de Leite Humano e 1 (7,7\%) em Centro de Terapia Intensiva Pediátrico.

Também participaram 20 (100\%) pais e/ou responsáveis, dos quais 18 (90\%) eram mães e 2 (10\%) pais, no qual 13 (65\%) tinham entre 20 a 30 anos e sete (35\%) tinham entre 31 a 40 anos. Quanto ao estado civil, 14 participantes (70\%) eram casados ou em união estável e 6 (30\%) solteiros. O grau de escolaridade dos mesmos são 1 (5\%) nenhuma escolaridade, 1 (5\%) ensino fundamental, 8 (40\%) ensino médio, 5 (25\%) ensino superior incompleto e 5 (25\%) superior completo. Sobre a ocupação, 5 (25\%) responsáveis possuíam carteira assinada, 4 (20\%) eram autônomos, 9 (45\%) do lar, 1 (5\%) não trabalhava e $1(5 \%)$ era estudante.

No que diz respeito aos seus filhos, $4(20 \%)$ crianças tinham idade entre 13 dias a 2 meses, $6(30 \%)$ entre 3 a 6 meses, $6(30 \%)$ entre 7 a 10 meses e $4(20 \%)$ entre 11 meses a 1 ano. E, 7 (35\%) permaneceram internados entre 2 a 20 dias, 5 (25\%) entre 21 a 40 dias, 4 (20\%) entre 41 a 60 dias e 4 (20\%) ficaram entre 61 a 90 dias internados. Destacando que um desses bebês ainda permanecia internado no momento da coleta dos dados. Além disso, em relação ao hospital de nascimento dos bebês 18 (90\%) eram públicos e 2 (10\%) privados.

O estudo obteve como resultado a construção e validação da cartilha educativa intitulada "Cuidados com o bebê prematuro", portanto, segue a descrição das etapas do estudo.

\section{Elaboração da cartilha}

Através da revisão de literatura, com base nos manuais do Ministério da Saúde, de artigos que abordavam questões sobre as dificuldades para os pais no cuidado gerais aos bebês prematuros, bem como no Calendário de Vacinação da Sociedade Brasileira e Imunizações (SBIm), foram avaliados assuntos para compor a cartilha.

Para a elaboração do manual educativo, tendo como objetivo facilitar a leitura e não se tornar cansativa para o público-alvo, foram priorizadas a leitura sucinta, linguagem corriqueira e compreensível, letras no tamanho visível e 22 ilustrações coloridas representando a miscigenação do Brasil, feito em design gráfico por um profissional especializado.

As etapas de tratamento das ilustrações foram feitas no programa Gimp 2.8 para que fossem aperfeiçoadas. Depois o material conseguinte foi exportado para o programa Corel Draw para transformação das ilustrações, adição de cores, luz e 
sombra, seguindo a paleta de cores da cartilha educativa. No fim, os arquivos originais foram salvos em formato ".CDR" (Corel Draw), as ilustrações exportadas para o formato PNG e os arquivos compilados em um documento "pdf".

No estilo da escrita foi utilizado fonte ObelixPro tamanho 40 para títulos, Humnst777 Blk BT tamanho 18 para textos e Lula Borges tamanho 24 para subtítulos.

Os conteúdos abordados na cartilha foram: processo de alta; a chegada em casa; as visitas ao bebê prematuro; o sono do bebê; higiene; troca de fralda; nutrição; pega do bebê ; oferta do leite no copinho; engasgo do bebê; remédio do prematuro; transporte do bebê prematuro; vacinas; sinais de alerta; e, acompanhamento.

\section{Validação do manual educativo com juízes especialistas e público-alvo}

A validação de materiais educativos é necessária para tornar o material mais oportuno, legítimo e completo. Essa etapa é de grande relevância para o aperfeiçoamento do material, nos quais, são sugeridas informações, substituição e reformulação de termos e ilustrações. A validação pelos juízes especialistas é fundamental para tornar essas tecnologias mais eficazes durante o processo de educação em saúde e de maior rigor científico. Indispensável também é a validação desse material com os representantes do público-alvo, sendo uma oportunidade em que se tem clareza do que realmente está faltando e o que não foi compreendido (Lima, Bezerra, Sousa, Rocha \& Oriá, 2017).

O IVC (Índice de Validação de Conteúdo Global) médio dos quatro itens analisados pelos juízes especialistas resultou em um IVC global com valor de 0,99 (99,0\%), demonstrados na Tabela 1.

Tabela 1 - Avaliação dos juízes quanto ao conteúdo, ilustrações, linguagem, composição visual e conteúdo da cartilha. Rio de Janeiro, RJ, Brasil, 2020. (IVC Global=99\%).

\begin{tabular}{|c|c|c|c|}
\hline Item & $\begin{array}{l}\text { Discordo muito/ } \\
\text { Discordo pouco }\end{array}$ & $\begin{array}{l}\text { Concordo pouco/ } \\
\text { Concordo muito }\end{array}$ & IVC do item \\
\hline \multicolumn{4}{|l|}{ 1. Ilustrações } \\
\hline $\begin{array}{l}\text { 1.1 As ilustrações são atrativas e claras para a } \\
\text { compreensão do conteúdo? }\end{array}$ & - & 13 & 1.0 \\
\hline $\begin{array}{l}\text { 1.2 A quantidade de imagens está adequada para o } \\
\text { conteúdo da cartilha? }\end{array}$ & - & 13 & 1.0 \\
\hline $\begin{array}{l}\text { 1.3 As ilustrações e os textos motivam o leitor para } \\
\text { compreensão do tema proposto? }\end{array}$ & - & 13 & 1.0 \\
\hline \multicolumn{4}{|l|}{ 2. Linguagem } \\
\hline 2.1 Linguagem clara e objetiva para o público alvo? & 1 & 12 & 0.92 \\
\hline \multicolumn{4}{|l|}{ 3. Composição visual } \\
\hline $\begin{array}{l}\text { 3.1 As cores que foram utilizadas nas imagens são } \\
\text { agradáveis? }\end{array}$ & - & 13 & 1.0 \\
\hline 3.2 Há uma distribuição lógica de temas? & - & 13 & 1.0 \\
\hline $\begin{array}{l}3.3 \text { O tipo de letra utilizado (formato e tamanho) } \\
\text { facilita a leitura? }\end{array}$ & - & 13 & 1.0 \\
\hline $\begin{array}{l}\text { 3.4 As cores aplicadas ao texto são pertinentes e } \\
\text { facilitadoras para a leitura? }\end{array}$ & - & 13 & 1.0 \\
\hline $\begin{array}{l}3.5 \text { A composição visual está atrativa e bem } \\
\text { organizada? }\end{array}$ & - & 13 & 1.0 \\
\hline \multicolumn{4}{|l|}{ 4. Conteúdo } \\
\hline $\begin{array}{l}4.1 \text { Os temas abordados são significativos e } \\
\text { relevantes? }\end{array}$ & - & 13 & 1.0 \\
\hline 4.2 As informações são atualizadas? & - & 13 & 1.0 \\
\hline $\begin{array}{l}\text { 4.3 Há aplicabilidade da cartilha educativa na } \\
\text { prática clínica do enfermeiro? }\end{array}$ & - & 13 & 1.0 \\
\hline IVC Global & & & 0.99 \\
\hline
\end{tabular}

Fonte: Autores (2020).

Vale ressaltar que mesmo com o IVC Global de 0,99 (99,0\%), algumas sugestões pertinentes dos juízes especialistas foram consideradas, produzindo alterações em títulos, frases e vocabulário, com o intuito de ser mais clara possível para o 
público alvo. Títulos, frases e palavras foram substituídos para: “Como devemos agir durante as visitas para o bebê?"; "É fundamental que os pais/responsáveis levem para as consultas de avaliação do crescimento e desenvolvimento e necessidades em saúde.”; “A enfermeira me disse que quando o bebê estiver acordado, deixe-o ficar em diferentes posições no berço e não apenas de barriga para cima; "Pulmãozinho do bebê"; "Pulso"; "Correntes de ar." Ademais, imagens foram acrescentadas e algumas modificadas, como é possível observar na Figura 1.

Figura 1 - Ilustrações da cartilha educativa antes (lado esquerdo) e após a validação com os juízes especialistas (lado direito).

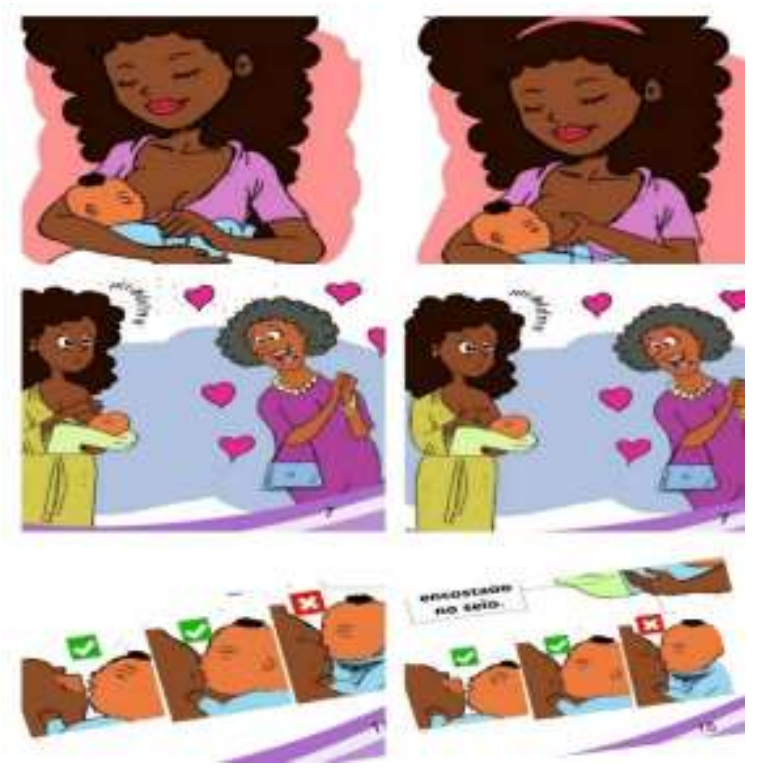

Fonte: Autores (2020).

Na página que se refere à amamentação foi colocado a mão da mãe em forma de "C" segurando corretamente a mama, sendo possível visualizar essa alteração no lado direito da Figura 2. Também na última página foi adicionada uma ilustração da família satisfeita com as orientações, visto que, antes não continha figura.

Na fase de validação com o público-alvo o IVC global obteve um valor de $0,98(98,0 \%)$, como observado na Tabela 2. 
Tabela 2 - Avaliação dos pais e responsáveis quanto ao conteúdo, ilustrações, linguagem, composição visual e conteúdo da cartilha. (IVC Global=99\%).

\begin{tabular}{|c|c|c|c|}
\hline Item & $\begin{array}{l}\text { Discordo muito/ } \\
\text { Discordo pouco }\end{array}$ & $\begin{array}{l}\text { Concordo pouco/ } \\
\text { Concordo muito }\end{array}$ & IVC do item \\
\hline \multicolumn{4}{|l|}{ 1. Ilustrações } \\
\hline $\begin{array}{l}\text { 1.1 As imagens são atrativas e claras para a } \\
\text { compreensão do conteúdo?" }\end{array}$ & - & 20 & 1.0 \\
\hline $\begin{array}{l}\text { 1.2 A quantidade de imagens está adequada para o } \\
\text { conteúdo da cartilha? }\end{array}$ & 1 & 19 & 0.95 \\
\hline \multicolumn{4}{|l|}{ 2. Linguagem } \\
\hline 2.1 Linguagem clara, de fácil leitura e compreensiva? & - & 20 & 1.0 \\
\hline 2.2 Sentiu-se estimulado a ler a cartilha? & - & 20 & 1.0 \\
\hline \multicolumn{4}{|l|}{ 3. Composição visual } \\
\hline $\begin{array}{l}\text { 3.1 As cores que foram utilizadas nas imagens são } \\
\text { agradáveis? }\end{array}$ & - & 20 & 1.0 \\
\hline $\begin{array}{l}\text { 3.2 As cores aplicadas ao texto são pertinentes e } \\
\text { facilitadoras para a leitura? }\end{array}$ & - & 20 & 1.0 \\
\hline $\begin{array}{l}3.3 \text { A composição visual está atrativa e bem } \\
\text { organizada? }\end{array}$ & - & 20 & 1.0 \\
\hline $\begin{array}{l}3.4 \mathrm{O} \text { tipo de letra (formato e tamanho) facilita a } \\
\text { leitura? }\end{array}$ & 1 & 19 & 0.95 \\
\hline \multicolumn{4}{|l|}{ 4. Conteúdo } \\
\hline $\begin{array}{l}\text { 4.1 As informações são úteis, esclarece possíveis } \\
\text { dúvidas? }\end{array}$ & - & 20 & 1.0 \\
\hline $\begin{array}{l}\text { 4.2 Todas as informações são necessárias e } \\
\text { relevantes? }\end{array}$ & - & 20 & 1.0 \\
\hline $\begin{array}{l}\text { 4.3 As informações mudaram a forma de como } \\
\text { cuidará do seu filho em casa? }\end{array}$ & 1 & 19 & 0.95 \\
\hline IVC Global & & & 0,98 \\
\hline
\end{tabular}

Fonte: Autores (2020).

Houve duas participantes que sugeriram modificações na parte de conteúdo e composição visual. Em relação ao conteúdo foi proposto inserir orientações sobre higiene íntima dos bebês e sobre a importância de mencionar que os medicamentos só devem ser oferecidos aos prematuros, se prescritos pelo médico. Foi considerado pertinente pelas autoras e então, acrescentado na página sobre troca de fraldas o trecho: "Higiene íntima menina: Com o algodão e água morna faça a limpeza em movimentos únicos de frente para trás (da vagina para o ânus). Não use o algodão mais de uma vez” e o trecho "Higiene íntima menino: Com algodão e água morna faça a limpeza no pênis, puxando a pele do prepúcio delicadamente. Com um novo algodão, limpe os testículos e a região ao redor do ânus, obedecendo sempre essa ordem". E também foi acrescentado na página "Remédio do bebê prematuro", o trecho "Só ofereça remédios ao seu bebê que forem prescritos pelo médico".

No que se refere à composição visual foi solicitado o aumento da fonte da letra da cartilha, entretanto, esta sugestão não foi ponderada, considerando-se que o IVC se mostra acima da média, sendo julgado satisfatório, de forma geral, por todos os outros participantes.

\section{Adequação da cartilha educativa}

Após os procedimentos de validação de ambas as partes, os itens apontados como insuficientes ou discordantes foram revisados e remodelados, com intuito de aprimorar e melhorar o manual educativo.

Foram consideradas algumas das sugestões propostas tais como: reestruturação de informações, a inserção de informações que os juízes especialistas julgaram necessárias, readequação de ilustrações e alteração de termos técnicos. Após a etapa de confirmação com os juízes especialistas, ocorreu o mesmo processo com o público-alvo e, apesar do IVC global 
indicar concordância de 98\%, foi julgado necessário fazer algumas das alterações sugeridas, na qual consistiram na adequação e substituição de termos, além do acréscimo de informações consideradas relevantes. Após as adequações finais, a versão final da cartilha educativa foi disponibilizada através de um link para dispositivos móveis, com a intenção de atingir um maior número de pessoas possível.

\section{Discussão}

Os materiais educativos são ferramentas importantes nos processos de ensino-aprendizagem e de educação em saúde, podendo ser adotadas como complemento ao diálogo. São capazes de potencializar as intervenções de saúde, o trabalho da equipe e ajudar na promoção da saúde dos sujeitos envolvidos, visto que permitem o acesso ao conteúdo de maneira facilitada, além de serem permanentes, já que estão disponíveis para consultas sempre que necessário (Lemos \& Veríssimo, 2020), conforme a atual cartilha que se encontra disponível de forma livre e gratuita.

$\mathrm{Na}$ etapa de validação os responsáveis relataram que não sabiam realizar os cuidados após a alta do recém-nascido da UTIN, descrevendo principalmente sentimentos como medo, desconhecimento e falta de habilidade. Diante da necessidade de um processo educativo-assistencial, a cartilha educativa surge como forma de elucidar e ratificar a necessidade de abordagem deste assunto (Silva, et al., 2020), corroborando os relatos dos responsáveis.

Estudo realizado em um hospital de Curitiba-PR demonstra que informações de saúde fornecidas por instrumentos educativos digitais podem colaborar para maior conhecimento dos pais favorecendo o desenvolvimento infantil, porém, afirma que as informações oferecidas durante a leitura devem ser complementares as dos profissionais de saúde e não, substitutas (Lima \& Mazza, 2019). Nessa mesma linha diretiva, a enfermagem utiliza-se de tecnologias educacionais para promover a educação em saúde como um meio para estimular a autonomia de seus clientes (Pinto, et al., 2018), o que corrobora com a motivação para a construção da presente cartilha educativa.

Outra pesquisa produzida no sul do Brasil, no ano de 2018, destacou novamente a relevância de materiais educativos digitais e impressos, na sensibilização de pacientes e familiares, ao avaliar durante um período de quatro anos a correlação entre a distribuição de folders e/ou cartazes de orientação sobre prevenção de quedas e a diminuição do índice de quedas (Luzia, et al., 2018). Acredita-se que a cartilha "Cuidados com o bebê prematuro" também possui potencial para instrumentalizar os familiares, na medida em que sua linguagem e imagens buscam estimular o desenvolvimento de cuidados de forma autônoma e segura.

Para que haja maior eficácia e adesão, é indispensável que a cartilha possua uma linguagem clara e acessível para todas as classes sociais e todos os graus de instrução. As imagens também são fundamentais para contribuir no entendimento das orientações escritas, além de estimularem a leitura. Além disso, a participação do público-alvo para o desenvolvimento e avaliação da cartilha demonstra sua importância nesse processo, não apenas como informantes e receptores dos resultados das investigações científicas, mas como participantes ativos na produção do conhecimento, conforme pressupõe as metodologias participativas de pesquisa e educação popular (Streck, 2016).

Assim, os pais/responsáveis avaliaram a presente cartilha "Cuidados com o bebê prematuro" de forma positiva, considerando importantes os tópicos abordados, a clareza das informações, formato e ilustrações adequadas. Esses achados confluem com o estudo sobre uma cartilha para prevenção do excesso ponderal em adolescentes, na qual o público-alvo também avaliou positivamente o material educativo, julgando como explicativo, interessante e motivador (Moura, et al., 2019). As avaliações de ambos os estudos indicam que os materiais podem ser utilizados entre os grupos-alvos.

Mesmo sabendo da importância do conhecimento das necessidades de aprendizagem dos pais de bebês prematuros, existe uma escassez de material referente à temática (Alcântara, et al., 2017; Silva, et al., 2020; Veronez \& Higarashi, 2016). 
Sendo, portanto, a limitação deste estudo a quantidade escassa de literaturas relacionadas ao tema, limitando uma discussão mais específica e comparativa dos achados.

Todavia, a validação do material teve um índice satisfatório e a pesquisa se demonstrou relevante por retratar a realidade dos pais com bebês prematuros, por isso, esta pesquisa serve como base para futuros trabalhos relacionados ao tema.

\section{Considerações Finais}

A cartilha educativa com orientações para "Cuidados com o bebê prematuro" foi construída e validada no que tange a aparência e conteúdo por juízes especialistas e pelo público-alvo, alcançando índices satisfatórios. Este material é uma tecnologia educativa que servirá de suporte e poderá ser utilizada por profissionais de saúde para que pais/responsáveis sanem suas dúvidas e dificuldades para com o cuidado de seus filhos após a alta hospitalar.

\section{Referências}

Alcântara K. L., Brito L. L. M. D. S., Costa D. V. da S., Façanha A. P. M., Ximenes L. B., \& Dodt R. C. M. . (2017). Orientações familiares necessárias para uma alta hospitalar segura do recém-nascido prematuro: revisão integrativaRev enferm UFPE, 11(2), 645-655.

Dias, I. M. A. V, Fialho, F. A., Silva, L. R., Santos, R. S., \& Salvador, M. (2015). Tecnologias aplicadas pela enfermagem no cuidado neonatal. Revista Baiana de Enfermagem, 29(1), 23-32.

Estevam D. C. M, \& Silva J. D. D. (2016). Visão das mães em relação ao cuidado com o recém-nascido após a alta da UTI neonatal. Saúde e pesquisa, 9(1), $15-24$.

Klock P., Buscher A., Erdmann A. L., Costa R., \& Santos S. V. (2019). Melhores práticas na gerência do cuidado de enfermagem neonatal. Texto \& Contexto - Enfermagem, 28, e20170157.

Lelis B. D. B, de Sousa M. I., de Mello D. F., Wernet M., Velozo A. B. F., \& Leite A. M. (2018). Acolhimento materno no contexto da prematuridade. Rev enferm UFPE on line, 12(6), 1563-1569.

Lemos R. A., \& Veríssimo M. D. L. Ó. R. (2020). Methodological strategies for the elaboration of educational material: focus on the promotion of preterm infants' development. Ciênc Saúde Colet, 25(2), 505-518.

Lima A. C. M. A. C. C,, Bezerra K. D. C, Sousa D. M. D. N, Rocha J. D. F, Oriá M. O. B. (2017) Construção e validação de cartilha para prevenção da transmissão vertical do HIV.. Acta Paul Enferm, 30(2), 181-189.

Lima V. F., \& Mazza V. D. A. Necessidades de informações das famílias sobre saúde/doença dos prematuros em unidade de terapia intensiva neonatal. (2019). Texto Context enferm, 28, e20170474.

Luzia M. D. F, Cassola T. P., Suzuki L. M., Dias V. L. M., de Pinho L. B., \& Lucena A. D. F. Incidence of falls and preventive actions in a University Hospital. (2018). Revista da Escola de Enfermagem da USP, 52, e03308.

Marski B de S. L., Custodio N., de Abreu F. C. P., de Melo D. F. \& Wernet M. Hospital discharge of premature newborns: the father's experience. (2016). Rev Bras Enferm, 69 (2), 221-228.

Medeiros R. K. D. S, Júnior Ferreira M. A., Pinto D. P. D. S. R, Vitor A. F., Santos V. E. P., \& Barichello E. Modelo de validação de conteúdo de Pasquali nas pesquisas em enfermagem. (2015). Rev Enf Ref, IV(4), 127-135.

Ministério da Saúde (Br). Atenção à saúde do recém-nascido: guia para os profissionais de saúde (Vol. 2). Brasília: Ministério da Saúde.

Moura J. R. A, da Silva K. C. B., Rocha A. D. E. S. D. H., dos Santos S. D., Amorim T. R. D. S., \& da Silva A. R. V. Construção e validação de cartilha para prevenção do excesso ponderal em adolescentes. (2019). Acta Paulista de Enfermagem, 32(4), 365-373.

Organización Mundial de la Salud. (2018). Nacimientos prematuros. https://www.who.int/es/news-room/fact-sheets/detail/preterm-birth

Pinto T D. R. C, de Castro D. S., Bringuente M. E. D. O, Sant'Anna H. C., Souza T. V., \& Primo C. C. Educational animation about home care with premature newborn infants. (2018). Rev Bras, 71(supl.4), 1604-1610.

Rodrigues B.C., Uema R. T. B., Rissi G. P., Felipin L. C. S., \& Higarashi I. H. Cuidado centrado na família e sua prática na unidade de terapia intensiva neonatal. (2019). Rev Rene, 20, e39767.

Silva F. V. D. R., Gomes T. D. O., Marta C. B., Araujo M. C., \& Braga E. D. S. Preparo dos pais de recém nascido pré-termo para alta hospitalar: proposta de um protocolo. (2020). Rev. Pesqui. (Univ. Fed. Estado Rio J., Online), 12, 420-426.

Silva I. O. A. M. D. , Aredes N. D. A., Bicalho M. B., Delácio N. C. B., Mazzo L. D. L., \& Fonseca L. M. M. Cartilha sobre o prematuro como tecnologia educacional para família: estudo quase experimental. (2018). Acta Paul Enferm, 31(4), 334-341. 
Research, Society and Development, v. 10, n. 15, e368101518007, 2021

(CC BY 4.0) | ISSN 2525-3409 | DOI: http://dx.doi.org/10.33448/rsd-v10i15.18007

Sousa C. S., Turrini R. N. T., \& Poveda V. B. Translation and adaptation of the instrument "suitability assessment of materials" (sam) into portuguese. (2015). Rev enferm UFPE, 9(5), 7854-7861.

Sousa S. C. D., Medino Y. M. S., Benevides K. G. C. B., Ibiapina A. D. S., \& Ataíde K. D. M. N. Fortalecimento do vínculo entre a família e o neonato prematuro. (2019). Rev enferm UFPE, 13(2), 298-306.

Streck D. R. Participatory research methodologies and popular education: reflections on quality criteria. (2016). Interface - Comunicação, Saúde, Educação, 20(58), 537-547.

Teixeira, J. A. M., Araujo, W. R. M., Maranhão, A. G. K., Cortez-Escalante, J. J., Rezende, L. F. M., \& Matijasevich, A. (2019). Mortalidade no primeiro dia de vida: tendências, causas de óbito e evitabilidade em oito Unidades da Federação brasileira, entre 2010 e 2015. Epidemiologia e Serviços de Saúde, 28(1), e2018132.

Veronez M., \& Higarashi I. H. Protocolo para a alta de bebê pré-termo: subsídios para a construção de uma proposta. (2016). Rev enferm UERJ, 24(3), 3-8.

Vinuto J. A amostragem em bola de neve na pesquisa qualitativa: um debate em aberto. Temáticas. (2014). Tematicas, 22(44), 203-220. 